\title{
Effect of proinflammatory interleukins on jejunal nutrient transport
}

\author{
J Hardin, K Kroeker, B Chung, D G Gall
}

\begin{abstract}
Aim-We examined the effect of proinflammatory and anti-inflammatory interleukins on jejunal nutrient transport and expression of the sodium-glucose linked cotransporter (SGLT-1).

Methods-3-O-methyl glucose and L-proline transport rates were examined in New Zealand White rabbit stripped, short circuited jejunal tissue. The effects of the proinflammatory cytokines interleukin (IL)-1 $\alpha$, IL-6, and IL-8, IL-1 $\alpha$ plus the specific IL-1 antagonist, IL-1ra, and the anti-inflammatory cytokine IL-10 were investigated. In separate experiments, passive tissue permeability was assessed and brush border SGLT-1 expression was measured by western blot in tissues exposed to proinflammatory interleukins.
\end{abstract}

Results-The proinflammatory interleukins IL-6, IL-1 $\alpha$, and IL-8 significantly increased glucose absorption compared with control levels. This increase in glucose absorption was due to an increase in mucosal to serosal flux. IL-1 $\alpha$ and IL-8 also significantly increased L-proline absorption due to an increase in absorptive flux. The anti-inflammatory IL-10 had no effect on glucose transport. The receptor antagonist IL-1 ra blocked the ability of IL-1 $\alpha$ to stimulate glucose transport. IL-8 had no effect on passive tissue permeability. SGLT-1 content did not differ in brush border membrane vesicles (BBMV) from control or interleukin treated tissue.

Conclusions-These findings suggest that intestinal inflammation and release of inflammatory mediators such as interleukins increase nutrient absorption in the gut. The increase in glucose transport does not appear to be due to changes in BBMV SGLT-1 content.

(Gut 2000;47:184-191)

Gastrointestinal

Research Group,

University of Calgary,

Calgary, Alberta,

Canada

J Hardin

K Kroeker

B Chung

D G Gall

Correspondence to:

Dr D G Gall, Faculty of

Medicine, Health Science

Centre, 3330 Hospital Drive

N W, Calgary, Alberta, T2N

$4 \mathrm{Nl}$, Canada. Email:

dggall@ucalgary.ca

Accepted for publication 22 February 2000

Interleukins (IL) are small soluble mediators that participate in a variety of physiological and pathological events. During inflammatory states interleukins regulate the intensity of the intestinal immune response and mediate altered physiology in response to the inflammatory process (for example, diarrhoea) either directly or via production of additional effector molecules. ${ }^{12}$ Interleukins are classified as proor anti-inflammatory based on their activity in promoting or inhibiting the inflammatory response. Interleukin- $1 \alpha$ (IL-1 $\alpha$ ), IL-6, and
IL-8 are among the best characterised proinflammatory interleukins while IL-10 has demonstrated anti-inflammatory activity. Aside from their role in the regulation of the immune cascade, interleukins have also been shown to modulate intestinal transport function. The proinflammatory IL- $1 \alpha$ has been reported to inhibit $\mathrm{Na}^{+}$and $\mathrm{Cl}^{-}$absorption in rabbit ileum ${ }^{3}$ while IL-1 $\beta$ stimulates anion secretion in chicken intestine. ${ }^{4}$ Conversely, the antiinflammatory cytokine IL-10 has been shown to stimulate net sodium and chloride absorption and inhibit stimulated chloride secretion in rat small intestine. ${ }^{5}$ IL-10 has also been shown to regulate epithelial permeability in addition to inhibiting stimulated chloride secretion in T84 cell monolayers. ${ }^{6}$ In contrast, IL-6 has no direct effect on chloride secretion in colonic HT29 cl 19A cells but reduces overall electrolyte transport following long term exposure. ${ }^{7}$

Systemic and acute inflammation is associated with an increase in whole body and tissue metabolic activity. ${ }^{89}$ A number of proinflammatory cytokines, notably IL- $1 \alpha$, have been reported to exert significant effects on central and peripheral glucose regulation which may be related to the increased metabolic demands of inflamed tissue. IL- $1 \alpha$ has been shown to act centrally to enhance whole body glucose metabolism ${ }^{10}$ and low intraperitoneal doses of IL- $1 \beta$ have been reported to affect glucose homeostasis in vivo by increasing insulin blood levels. ${ }^{11}$ IL-3 promoted glucose transport in a bone marrow derived cell line ${ }^{12}$ and IL- $1 \alpha$ has been shown to stimulate glycolysis and glucose uptake in human dermal fibroblasts, ${ }^{13}$ and glucose uptake and phosphorylation in murine astrocytes. ${ }^{14}$ These findings suggest a role for proinflammatory interleukins in the regulation of glucose transport in response to inflammatory events.

We hypothesised that because of increased metabolic demands, proinflammatory cytokines upregulate intestinal nutrient absorption. Therefore, the aim of this study was to examine the role of pro- and anti-inflammatory cytokines in the regulation of intestinal nutrient transport.
Abbreviations used in this paper: TTBS, $0.05 \%$ Tween 20 Tris buffered saline; BSA, bovine serum albumin; BBMV, brush border membrane vesicle; EDTA, ethylene diaminetetraacetic acid; SGLT-1, sodium-glucose linked cotransporter; IL, interleukin; $P D$, potential difference; $I_{\mathrm{sc}}$, short circuit current; $\mathrm{G}$, conductance; $\mathrm{J}_{\mathrm{ms}}$, mucosal to serosal flux; $\mathrm{J}_{\mathrm{sm}}$, serosal to mucosal flux; $\mathrm{J}_{\text {net }}$, net flux; SDS/PAGE, sodium dodecyl sulphate-polyacrylamide gel electrophoresis. 
Methods and materials

New Zealand White rabbits $(800-1200 \mathrm{~g})$ were used for the study. Animals were killed after a 24 hour fast and a $10 \mathrm{~cm}$ segment of jejunum, beginning $5 \mathrm{~cm}$ distal to the ligament of Treitz, was removed. The lumenal contents of the segment were flushed with cold Kreb's solution and the tissue was stripped of its muscle and serosa. Four adjacent pieces of tissue that did not contain Peyer's patches were mounted in Ussing-type chambers with apertures of $0.4 \mathrm{~cm}^{2}$. Mucosal and serosal surfaces were bathed with $10 \mathrm{ml}$ of oxygenated Kreb's buffer (120.3 $\mathrm{mM} \mathrm{NaCl}, 5.9 \mathrm{mM} \mathrm{KCl}, 2.5 \mathrm{mM}$ $\mathrm{CaCl}_{2}, 1.2 \mathrm{mM} \mathrm{MgCl}, 15.4 \mathrm{mM} \mathrm{NaHCO}$, $1.2 \mathrm{mM} \mathrm{NaH}_{2} \mathrm{PO}_{4}, \mathrm{pH} 7.35$ at $37^{\circ} \mathrm{C}$ ). In addition, the serosal buffer contained $10 \mathrm{mM}$ glucose to provide metabolic energy to the tissue and the mucosal buffer contained $10 \mathrm{mM}$ mannitol to osmotically balance the bathing solutions. The spontaneous potential difference (PD) was determined and the tissue clamped at zero voltage by introducing an appropriate short circuit current $\left(\mathrm{I}_{\mathrm{sc}}\right)$ with an automatic voltage clamp (World Precision Instruments, Narco Scientific, Downsview, Canada). $I_{s c}$ was continuously monitored and PD measured by briefly removing the voltage clamp for $2-3$ seconds every five minutes. PD is expressed as millivolts $(\mathrm{mV}), I_{s c}$ as $\mu \mathrm{Eq} / \mathrm{cm}^{2} /$ hour, and conductance as millisiemens $/ \mathrm{cm}^{2}$ $\left(\mathrm{mS} / \mathrm{cm}^{2}\right)$. Tissue pairs were discarded if conductance varied by $>25 \%$.

NUTRIENT TRANSPORT

Experiments examined the effect of pro- and anti-inflammatory interleukins on in vitro unidirectional and net jejunal 3-O-methyl glucose and L-proline fluxes. 3-O-methyl glucose (20 $\mathrm{mM}$ ) (Aldrich, Milwaukee, Wisconsin, USA) was added to both mucosal and serosal bathing solutions in all chamber experiments except those examining L-proline and L-glucose transport. In experiments assessing glucose transport, $10 \mu \mathrm{Ci}$ of $\left[{ }^{3} \mathrm{H}\right] 3-O-$ methyl glucose were added to either the serosal or mucosal side immediately after mounting. In separate experiments to assess amino acid transport, $5 \mathrm{mM}$ of L-proline were added to the mucosal and serosal bathing solutions and, immediately after mounting, $10 \mu \mathrm{Ci}$ of L- $\left[{ }^{3} \mathrm{H}\right]$-proline were added to either the serosal or mucosal side. L-Glucose transport was assessed following addition of $20 \mathrm{mM}$ of L-glucose to both surfaces and addition of $10 \mu \mathrm{Ci}$ of $\left[{ }^{3} \mathrm{H}\right]-\mathrm{L}-$ glucose to the mucosal or serosal bathing solution. Cr-EDTA permeability was assessed following addition of $10 \mu \mathrm{Ci}$ of $\left[{ }^{51} \mathrm{Cr}\right]$-EDTA to the mucosal or serosal reservoir. After a 20 minute equilibration period, unidirectional mucosa to serosa $\left(J_{\mathrm{ms}}\right)$, serosa to mucosa $\left(\mathrm{J}_{\mathrm{sm}}\right)$, and net $\left(\mathrm{J}_{\text {net }}\right)$ fluxes were determined by measuring one overall 15 minute flux and three consecutive five minute fluxes to ensure steady state conditions. In studies assessing 3-Omethyl glucose transport, IL- $1 \alpha$ (at 5,10 , or 50 $\mathrm{ng} / \mathrm{ml} ; 0.28 \mathrm{nM}, 0.56 \mathrm{nM}$, or $2.8 \mathrm{nM}$ ), IL- $1 \alpha+\mathrm{IL}-1 \mathrm{ra}$ (at 10 and $100 \mathrm{ng} / \mathrm{ml}$, respectively; 0.56 and $5.9 \mathrm{nM})$, IL-1ra alone (100 $\mathrm{ng} / \mathrm{ml} ; 5.9 \mathrm{nM})$, IL-6 (10 ng/ml; $0.49 \mathrm{nM}$ ),
IL-8 (at 10 or $100 \mathrm{ng} / \mathrm{ml} ; 1.2$ or $12.5 \mathrm{nM}$ ), or IL-10 $(10 \mathrm{ng} / \mathrm{ml} ; 0.54 \mathrm{nM})$ was then added to the serosal surface. In the proline studies, 5 or $50 \mathrm{ng} / \mathrm{ml}$ of IL- $1 \alpha$ or $100 \mathrm{ng} / \mathrm{ml}$ of IL- 8 were added to the serosal surface. The tissue was then allowed to equilibrate for an additional five minutes after which a further three, five minute fluxes and overall 15 minute flux were determined. Subsequent studies examining the mechanisms involved in the effect of proinflammatory interleukins on nutrient transport used IL-8 at a concentration of $100 \mathrm{ng} / \mathrm{ml}$ as this produced the greatest increase in net 3-O-methyl glucose transport seen in the study. In separate experiments, the role of the enteric nervous system in mediating the effect of the proinflammatory interleukins on nutrient transport was examined by addition of $5 \times 10^{-7} \mathrm{M}$ tetrodotoxin (TTX; Sigma, St Louis, Missouri, USA) to the serosal surface immediately after mounting of the tissue. After a 20 minute equilibration period, unidirectional mucosa to serosa, serosa to mucosa, and net fluxes were determined over an initial 15 minute basal period and then for a further 15 minute period, five minutes following addition of $100 \mathrm{ng} / \mathrm{ml}$ of IL-8 to the serosal bathing solution. Neural blockade was confirmed by transmural field stimulation at the end of each experiment. All interleukins and the IL-1 receptor antagonist (IL-1 ra) were obtained from R+D Systems (Minneapolis, Minnesota, USA). Fluxes were calculated as previously described ${ }^{15}$ and expressed as $\mu \mathrm{Eq} / \mathrm{cm}^{2} /$ hour. $\left[{ }^{51} \mathrm{Cr}\right]$-EDTA movement is expressed as percentage of total "hot" side radioactivity crossing to the "cold" side per five minute period.

\section{$\mathrm{Na}^{+} / \mathrm{K}^{+}$ATPase ACTIVITY}

In separate experiments, stripped jejunal tissue was mounted in short circuited Ussing chambers as described above. After a 20 minute equilibration period and a subsequent 15 minute basal period, $100 \mathrm{ng} / \mathrm{ml}$ of IL- 8 was added to the serosal surface in two of the chambers and vehicle added to the remaining two chambers. The tissue was maintained for a further 20 minutes and then the chambers were broken down, tissue removed, and the mucosa exposed to the Ussing chamber lumen, scraped, homogenised in $2.5 \mathrm{mM}$ EDTA, flash frozen, and stored at $-70^{\circ} \mathrm{C}$ until the day of assay. Mucosal homogenates were assessed for protein content by the method of Lowry and colleagues. ${ }^{16}$ Mucosal $\mathrm{Na}^{+} / \mathrm{K}^{+}$ATPase activity was measured by the method of Kelly and colleagues $^{17}$ and values are reported as $\mathrm{U} / \mathrm{mg}$ protein.

\section{SGLT-1 EXPRESSION}

Jejunal tissue was removed, stripped, and mounted into short circuited Ussing chambers as described above. To maximise mucosal yield, large $1.86 \mathrm{~cm}^{2}$ aperture chambers were used. The tissues were paired and, in separate experiments, after a 20 minute equilibration period either IL-8 (100 ng/ml) or IL-6 (10 $\mathrm{ng} / \mathrm{ml}$ ) was added to the serosal surface in two of the four chambers. After 15 minutes the tissue was removed and mucosa scraped for 
preparation of brush border membrane vesicles (BBMV).

Brush border membrane vesicle isolation

BBMV were prepared by a calcium chloride precipitation method as previously described. ${ }^{18}$ BBMV were stored in liquid nitrogen until the day of assay. All measurements were normalised to membrane protein content as determined by the method of Lowry and colleagues. ${ }^{16}$ Purity and basolateral contamination of membranes was assessed by determining sucrase activity by the method of Dalquist, ${ }^{19}$ and $\mathrm{Na}^{+} / \mathrm{K}^{+}$ATPase activity by the method of Kelly and colleagues, ${ }^{17}$ in both the initial mucosal homogenate and microvillus membrane preparations.

\section{Western blot}

BBMV were separated on $8 \%$ denaturing sodium dodecyl sulphate-polyacrylamide gel electrophoresis (SDS/PAGE) mini-gels as previously described. ${ }^{20}$ Briefly, BBMV were diluted $1: 3$ in $2 \times$ Laemmli SDS protein sample buffer (S-3401, Sigma, St Louis, Missouri, USA) and boiled at $100^{\circ} \mathrm{C}$ for five minutes to denature and linearise the proteins. BBMV were then loaded onto $8 \%$ SDS mini-gels (25 $\mu \mathrm{g}$ protein/lane) together with a high range molecular weight marker set (V-5251, Promega, Madison, Wisconsin, USA); the lanes were separated using a constant current. Separated proteins were transferred overnight onto nitrocellulose paper (Trans-Blot Transfer Medium, $0.45 \mu \mathrm{m}$, Bio-rad, Mississauga, Ontario, Canada) with low ionic transfer buffer at constant current. Finished blots were rinsed in phosphate buffered saline, blotted dry on filter paper, and stored at $-20^{\circ} \mathrm{C}$ until later use.

Blots were probed with a polyclonal antibody directed against residues 402-420 of the rabbit SGLT-1 sequence ${ }^{21}$ (8327-1109, Cedarlane Laboratories Ltd., Hornby, Ontario, Canada). Immunoreactive bands were enhanced using a modification of the immunogold-silver technique, as described by Fowler. ${ }^{22}$ Briefly, blots were thawed, rehydrated with $0.05 \%$ Tween 20 Tris buffered saline (TTBS), and then blocked with $5 \% \mathrm{w} / \mathrm{v}$ bovine serum albumin (BSA) in TTBS for one hour followed by three 10 minute washes with TTBS. Purified anti-SGLT-1 antibody was diluted $1: 100 \mathrm{v} / \mathrm{v}$ in $1 \% \mathrm{BSA} / \mathrm{TTBS}$ and incubated with the blots for four hours. Blots were then given three more 10 minute washes in TTBS and probed with a secondary antibody ( $\alpha-$ rabbit IgG conjugated to $10 \mathrm{~nm}$ gold, G-7402, Sigma, St Louis, Missouri, USA) diluted 1:200 $\mathrm{v} / \mathrm{v}$ in gelatin buffer $(0.1 \% \mathrm{BSA} / 0.4 \%$ gelatin/ TTBS) for 1.5 hours. Blots were washed three more times with TTBS and once with $\mathrm{ddH}_{2} \mathrm{O}$ and then incubated with silver enhancer (RPN 492, Cedarlane Laboratories Ltd) for 30-45 minutes to amplify the gold signal. Immunoreactive bands were analysed by two dimensional scanning densitometry with incident illumination on a Scanalytics SCPI Masterscan densitometer, using Camscan (Scanalytics). The image was then analysed by RFLPScan 1.01 and expressed as units of integrated optical density.

STATISTICAL ANALYSIS

Data are expressed as mean (SEM) and statistical analyses were performed using the paired $t$ test. An associated probability of $\leqslant 5 \%$ was considered significant.

\section{Results}

3-O-METHYL GLUCOSE TRANSPORT

In the absence of drugs or interleukins, the mucosal to serosal, serosal to mucosal, and net fluxes of 3-O-methyl glucose did not differ between the first and second study periods. As shown in table 1 , the proinflammatory interleukin IL- $1 \alpha$ at concentrations of 5,10 , and $50 \mathrm{ng} / \mathrm{ml}$ significantly increased net jejunal 3-O-methyl glucose uptake. The increase in net 3-O-methyl glucose uptake was due to a significant increase in mucosal to serosal flux. IL-1 $\alpha$ also resulted in a small but significant increase in serosal to mucosal flux at concentrations of 5 and $50 \mathrm{ng} / \mathrm{ml}$. The delta increase, or difference, in net 3-O-methyl glucose uptake was maximal at a concentration of $5 \mathrm{ng} / \mathrm{ml}$ IL-1 $\alpha$. The delta increase in net 3-O-methyl

Table 1 Effect of IL-1a and IL-1 ra on jejunal 3-O-methyl glucose transport

\begin{tabular}{|c|c|c|c|c|c|c|}
\hline & $\begin{array}{l}\mathcal{F}_{m s} \\
\left(\mu E q / \mathrm{cm}^{2} / h\right)\end{array}$ & $\begin{array}{l}\mathcal{f}_{s m} \\
\left(\mu E q / \mathrm{cm}^{2} / h\right)\end{array}$ & 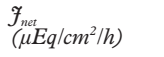 & $\begin{array}{l}P D \\
(m V)\end{array}$ & $\begin{array}{l}I_{s c} \\
\left(\mu \mathrm{A} / \mathrm{cm}^{2}\right)\end{array}$ & $\begin{array}{l}G \\
\left(\mathrm{mS} / \mathrm{cm}^{2}\right)\end{array}$ \\
\hline Basal period 1 & $0.80(0.09)$ & $0.22(0.02)$ & $0.58(0.10)$ & $3.3(0.2)$ & $38(5)$ & $29(3)$ \\
\hline $\begin{array}{l}\text { Control period } 2 \\
(\mathrm{n}=8)\end{array}$ & $\begin{array}{l}0.89(0.08) \\
\Delta=0.10(0.04)\end{array}$ & $\begin{array}{l}0.27(0.02) \\
\Delta=0.05(0.03)\end{array}$ & $\begin{array}{l}0.62(0.08) \\
\Delta=0.04(0.04)\end{array}$ & $3.0(0.2)$ & $34(5)^{a}$ & $28(3)$ \\
\hline Basal & $0.74(0.07)$ & $0.13(0.01)$ & $0.62(0.07)$ & $3.4(0.2)$ & $34(3)$ & $26(2)$ \\
\hline $\begin{array}{c}\mathrm{IL}-1 \alpha(5 \mathrm{ng} / \mathrm{ml}) \\
(\mathrm{n}=5)\end{array}$ & $\begin{array}{l}0.97(0.11)^{\mathrm{a}} \\
\Delta=0.22(0.05)\end{array}$ & $\begin{array}{l}0.19(0.01)^{\mathrm{a}} \\
\Delta=0.06(0.01)\end{array}$ & $\begin{array}{l}0.78(0.1)^{\mathrm{a}} \\
\Delta=0.17(0.05)\end{array}$ & $3.0(0.2)$ & 30 (3) & $26(2)$ \\
\hline Basal & $0.87(0.05)$ & $0.15(0.01)$ & $0.72(0.06)$ & $3.2(0.2)$ & $42(4)$ & $34(4)$ \\
\hline $\begin{array}{c}\mathrm{IL}-1 \alpha(10 \mathrm{ng} / \mathrm{ml}) \\
(\mathrm{n}=5)\end{array}$ & $\begin{array}{l}1.14(0.06)^{\mathrm{b}} \\
\Delta=0.27(0.04)\end{array}$ & $\begin{array}{l}0.2(0.03) \\
\Delta=0.05(0.02)\end{array}$ & $\begin{array}{l}0.95(0.06)^{\mathrm{a}} \\
\Delta=0.22(0.06)\end{array}$ & $2.8(0.1)^{\mathrm{a}}$ & 40 (3) & $36(4)$ \\
\hline Basal & $0.88(0.08)$ & $0.18(0.03)$ & $0.70(0.09)$ & $3.4(0.3)$ & $40(3)$ & $30(2)$ \\
\hline $\begin{array}{l}\mathrm{IL}-1 \alpha(50 \mathrm{ng} / \mathrm{ml}) \\
(\mathrm{n}=6)\end{array}$ & $\begin{array}{l}1.16(0.14)^{\mathrm{a}} \\
\Delta=0.28(0.08)\end{array}$ & $\begin{array}{l}0.26(0.02)^{\mathrm{a}} \\
\Delta=0.07(0.02)\end{array}$ & $\begin{array}{l}0.91(0.13)^{\mathrm{a}} \\
\Delta=0.21(0.07)\end{array}$ & $3.0(0.3)^{\mathrm{b}}$ & $35(2)^{b}$ & $29(2)$ \\
\hline Basal & $0.78(0.1)$ & $0.12(0.01)$ & $0.66(0.1)$ & $3.1(0.3)$ & $34(4)$ & $28(3)$ \\
\hline $\begin{array}{l}\text { IL-1 } \alpha(10 \mathrm{ng} / \mathrm{ml})+ \\
\text { IL-1 } 1 \mathrm{ra}(100 \mathrm{ng} / \mathrm{ml}) \\
(\mathrm{n}=5)\end{array}$ & $\begin{array}{l}0.91(0.14) \\
\Delta=0.13(0.06)\end{array}$ & $\begin{array}{l}0.15(0.02) \\
\Delta=0.03(0.01)\end{array}$ & $\begin{array}{l}0.77(0.15) \\
\Delta=0.10(0.06)\end{array}$ & $2.7(0.2)^{\mathrm{a}}$ & $29(4)^{\mathrm{a}}$ & 27 (3) \\
\hline Basal & $0.79(0.04)$ & $0.17(0.01)$ & $0.62(0.05)$ & $3.2(0.4)$ & $35(2)$ & $27(4)$ \\
\hline $\begin{array}{l}\mathrm{IL}-1 \mathrm{ra}(100 \mathrm{ng} / \mathrm{ml}) \\
(\mathrm{n}=4)\end{array}$ & $\begin{array}{l}0.89(0.04) \\
\Delta=0.10(0.01)\end{array}$ & $\begin{array}{l}0.19(0.03) \\
\Delta=0.02(0.03)\end{array}$ & $\begin{array}{l}0.70(0.04) \\
\Delta=0.08(0.03)\end{array}$ & $2.9(0.1)$ & $31(2)$ & $26(2)$ \\
\hline
\end{tabular}

Data are mean (SEM).

$\mathrm{J}_{\mathrm{ms}}$, mucosal to serosal flux; $\mathrm{J}_{\mathrm{sm}}$, serosal to mucosal flux; $\mathrm{J}_{\mathrm{net}}$, net flux; PD, potential difference; $\mathrm{I}_{\mathrm{sc}}$, short circuit current; $\mathrm{G}$, conductance. ${ }^{\mathrm{a}} \mathrm{p}<0.05,{ }^{\mathrm{b}} \mathrm{p}<0.01$ compared with basal period. 
Table 2 Effect of IL-6, IL-8, and IL-10 on jejunal 3-O-methyl glucose transport

\begin{tabular}{|c|c|c|c|c|c|c|}
\hline & $\begin{array}{l}\mathcal{F}_{m s} \\
\left(\mu E q / \mathrm{cm}^{2} / h\right)\end{array}$ & $\begin{array}{l}\mathcal{F}_{s m} \\
\left(\mu E q / \mathrm{cm}^{2} / h\right)\end{array}$ & $\begin{array}{l}\mathcal{F}_{\text {net }} \\
\left(\mu E q / \mathrm{cm}^{2} / h\right)\end{array}$ & $\begin{array}{l}P D \\
(m V)\end{array}$ & $\begin{array}{l}I_{s c} \\
\left(\mu \mathrm{A} / \mathrm{cm}^{2}\right)\end{array}$ & $\begin{array}{l}G \\
\left(\mathrm{mS} / \mathrm{cm}^{2}\right)\end{array}$ \\
\hline Basal & $0.98(0.11)$ & $0.20(0.02)$ & $0.78(0.10)$ & $3.3(0.2)$ & $33(3)$ & $26(2)$ \\
\hline $\begin{array}{l}\mathrm{IL}-6(10 \mathrm{ng} / \mathrm{ml}) \\
(\mathrm{n}=6)\end{array}$ & $\begin{array}{l}1.21(0.1)^{\mathrm{b}} \\
\Delta=0.24(0.05)\end{array}$ & $\begin{array}{l}0.26(0.02)^{\mathrm{b}} \\
\Delta=0.06(0.01)\end{array}$ & $\begin{array}{l}0.94(0.06)^{\mathrm{a}} \\
\Delta=0.17(0.05)\end{array}$ & $3.0(0.2)^{\mathrm{b}}$ & $29(2)$ & $25(2)$ \\
\hline Basal & $0.92(0.09)$ & $0.19(0.02)$ & $0.74(0.09)$ & $3.5(0.2)$ & $42(4)$ & $31(4)$ \\
\hline $\begin{array}{l}\mathrm{IL}-8(10 \mathrm{ng} / \mathrm{ml}) \\
(\mathrm{n}=5)\end{array}$ & $\begin{array}{l}1.23(0.15)^{\mathrm{b}} \\
\Delta=0.31(0.06)\end{array}$ & $\begin{array}{l}0.26(0.02)^{\mathrm{b}} \\
\Delta=0.08(0.01)\end{array}$ & $\begin{array}{l}0.97(0.15)^{\mathrm{a}} \\
\Delta=0.23(0.06)\end{array}$ & $3.2(0.2)^{\mathrm{a}}$ & $39(3)$ & $31(4)$ \\
\hline Basal & $0.85(0.19)$ & $0.18(0.02)$ & $0.68(0.17)$ & $3.3(0.3)$ & $32(5)$ & $25(2)$ \\
\hline $\begin{array}{l}\mathrm{IL}-8(100 \mathrm{ng} / \mathrm{ml}) \\
\quad(\mathrm{n}=4)\end{array}$ & $\begin{array}{l}1.14(0.18)^{\mathrm{b}} \\
\Delta=0.29(0.03)\end{array}$ & $\begin{array}{l}0.20(0.01) \\
\Delta=0.02(0.01)\end{array}$ & $\begin{array}{l}0.94(0.17)^{\mathrm{b}} \\
\Delta=0.26(0.03)\end{array}$ & $3.0(0.3)^{\mathrm{b}}$ & $29(4)$ & $24(2)$ \\
\hline Basal & $0.91(0.15)$ & $0.20(0.04)$ & $0.70(0.15)$ & $3.2(0.2)$ & $35(3)$ & $28(2)$ \\
\hline $\begin{array}{l}\mathrm{IL}-10(10 \mathrm{ng} / \mathrm{ml}) \\
\quad(\mathrm{n}=5)\end{array}$ & $\begin{array}{l}1.06(0.14) \\
\Delta=0.16(0.10)\end{array}$ & $\begin{array}{l}0.27(0.03)^{\mathrm{a}} \\
\Delta=0.07(0.02)\end{array}$ & $\begin{array}{l}0.79(0.14) \\
\Delta=0.09(0.08)\end{array}$ & $2.9(0.2)$ & $32(3)^{a}$ & $27(2)$ \\
\hline
\end{tabular}

Data are mean (SEM).

$\mathrm{J}_{\mathrm{ms}}$, mucosal to serosal flux; $\mathrm{J}_{\mathrm{sm}}$, serosal to mucosal flux; $\mathrm{J}_{\mathrm{net}}$, net flux; PD, potential difference; $\mathrm{I}_{\mathrm{sc}}$, short circuit current; $\mathrm{G}$, conductance. ${ }^{\mathrm{a} p}<0.05,{ }^{\mathrm{b}} \mathrm{p}<0.01$ compared with basal period.

Table 3 Effect of neural blockade on IL-8 stimulated 3-O-methyl glucose transport

\begin{tabular}{|c|c|c|c|c|c|c|}
\hline & $\begin{array}{l}\mathcal{F}_{m s} \\
\left(\mu E q / \mathrm{cm}^{2} / h\right)\end{array}$ & $\begin{array}{l}\mathcal{F}_{s m} \\
\left(\mu \mathrm{Eq} / \mathrm{cm}^{2} / \mathrm{h}\right)\end{array}$ & $\begin{array}{l}\mathcal{F}_{\text {net }} \\
\left(\mu E q / \mathrm{cm}^{2} / h\right)\end{array}$ & $\begin{array}{l}P D \\
(m V)\end{array}$ & $I_{s c}\left(\mu \mathrm{A} / \mathrm{cm}^{2}\right)$ & $\begin{array}{l}G \\
\left(\mathrm{mS} / \mathrm{cm}^{2}\right)\end{array}$ \\
\hline Basal & $0.83(0.12)$ & $0.24(0.03)$ & $0.59(0.12)$ & $2.7(0.2)$ & $30(2)$ & $28(2)$ \\
\hline $\begin{array}{l}\mathrm{IL}-8(100 \mathrm{ng} / \mathrm{ml}) \\
\quad(\mathrm{n}=5)\end{array}$ & $\begin{array}{l}1.25(0.13)^{\mathrm{a}} \\
\Delta=0.42(0.12)\end{array}$ & $\begin{array}{l}0.25(0.04) \\
\Delta=0.01(0.04)\end{array}$ & $\begin{array}{l}0.99(0.14)^{\mathrm{a}} \\
\Delta=0.40(0.15)\end{array}$ & $2.2(0.2)^{\mathrm{b}}$ & $24(1)^{b}$ & $27(2)$ \\
\hline
\end{tabular}

Data are mean (SEM).

$\mathrm{J}_{\mathrm{ms}}$, mucosal to serosal flux; $\mathrm{J}_{\mathrm{sm}}$, serosal to mucosal flux; $\mathrm{J}_{\text {net }}$, net flux; PD, potential difference; $\mathrm{I}_{\mathrm{sc}}$, short circuit current; $\mathrm{G}$, conductance. ${ }^{\mathrm{a}} \mathrm{p}<0.05,{ }^{\mathrm{b}} \mathrm{p}<0.01$ compared with basal period.

TTX $5 \times 10^{-7} \mathrm{M}$ was added to the serosal reservoir of all chambers immediately after mounting of the tissue.

glucose uptake did not differ between experiments performed with 5,10 , or $50 \mathrm{ng} / \mathrm{ml}$ of IL- $1 \alpha$. The effect of IL- $1 \alpha$ on 3-O-methyl glucose transport was abolished by the specific receptor antagonist IL-1 ra. In vitro 3-O-methyl glucose $\mathrm{J}_{\mathrm{ms}}, \mathrm{J}_{\mathrm{sm}}$, and $\mathrm{J}_{\text {net }}$ fluxes did not differ between basal and stimulated periods when IL- $1 \alpha$ was applied at a concentration of $10 \mathrm{ng} / \mathrm{ml}$ in the presence of $100 \mathrm{ng} / \mathrm{ml}$ of IL-1 ra. IL-1 ra alone at a concentration of 100 $\mathrm{ng} / \mathrm{ml}$ had no effect on $\mathrm{J}_{\mathrm{ms}}, \mathrm{J}_{\mathrm{sm}}$, or $\mathrm{J}_{\text {net }}$ 3-O-methyl glucose transport. Table 2 shows the effect of IL-6, IL-8, and IL-10 on in vitro 3-O-methyl glucose transport. The proinflammatory interleukins IL-6 $(10 \mathrm{ng} / \mathrm{ml})$ and IL-8 (at 10 and $100 \mathrm{ng} / \mathrm{ml}$ ) increased net 3-O-methyl glucose uptake. As was the case for IL- $1 \alpha$, the increase in net 3-O-methyl glucose uptake following application of IL-6 or IL-8 was due to a significant increase in mucosal to serosal flux of 3-Omethyl glucose. The serosal to mucosal flux of 3-O-methyl glucose also slightly but significantly increased in the presence of $10 \mathrm{ng} / \mathrm{ml}$ of IL-6 and IL-8. In contrast, the antiinflammatory interleukin IL-10 at a concentration of $10 \mathrm{ng} / \mathrm{ml}$ had no effect on mucosal to serosal or net flux of 3-O-methyl glucose.
Application of IL-10 to chambered jejunal tissue resulted in a significant increase in the serosal to mucosal flux of 3-O-methyl glucose. As shown in table 3, neural blockade had no effect on the increase in 3-O-methyl glucose transport observed after addition of $100 \mathrm{ng} / \mathrm{ml}$ of IL- 8 to the serosal surface.

\section{PROLINE TRANSPORT}

Further experiments were performed with the proinflammatory interleukins IL- $1 \alpha$ and IL- 8 to determine if the effect of these mediators was specific for 3-O-methyl glucose uptake or if they produced a more generalised elevation of nutrient uptake. Table 4 shows the effect of IL- $1 \alpha$ (at 5 and $50 \mathrm{ng} / \mathrm{ml}$ ) and IL-8 (100 $\mathrm{ng} / \mathrm{ml}$ ) on in vitro L-proline transport. At a concentration of $5 \mathrm{ng} / \mathrm{ml}$, IL- $1 \alpha$ had no effect on mucosal to serosal, serosal to mucosal, or net flux of L-proline. At a concentration of $50 \mathrm{ng} / \mathrm{ml}$, IL- $1 \alpha$ caused a small but significant increase in mucosal to serosal and net flux of L-proline. Similarly, IL-8 (100 ng/ml) also stimulated an increase in net transport of L-proline due to an increase in mucosal to serosal flux. IL-8 also caused a small but

Table 4 Effect of IL-1a and IL-8 on jejunal proline transport

\begin{tabular}{|c|c|c|c|c|c|c|}
\hline & $\begin{array}{l}\mathcal{J}_{m s} \\
\left(\mu E q / \mathrm{cm}^{2} / h\right)\end{array}$ & $\begin{array}{l}\mathcal{F}_{s m} \\
\left(\mu E q / \mathrm{cm}^{2} / h\right)\end{array}$ & $\begin{array}{l}\mathcal{F}_{\text {net }} \\
\left(\mu E q / \mathrm{cm}^{2} / h\right)\end{array}$ & $\begin{array}{l}P D \\
(m V)\end{array}$ & $I_{s c}\left(\mu \mathrm{A} / \mathrm{cm}^{2}\right)$ & $\begin{array}{l}G \\
\left(\mathrm{mS} / \mathrm{cm}^{2}\right)\end{array}$ \\
\hline Basal & $0.76(0.11)$ & $0.15(0.01)$ & $0.61(0.1)$ & $2.5(0.3)$ & $28(5)$ & $27(3)$ \\
\hline $\begin{array}{l}\mathrm{IL}-1 \alpha(5 \mathrm{ng} / \mathrm{ml}) \\
(\mathrm{n}=4)\end{array}$ & $\begin{array}{l}0.80(0.13) \\
\Delta=0.04(0.02)\end{array}$ & $\begin{array}{l}0.17(0.01) \\
\Delta=0.02(0.01)\end{array}$ & $\begin{array}{l}0.63(0.13) \\
\Delta=0.02(0.03)\end{array}$ & $2.5(0.3)$ & $27(4)$ & $26(3)$ \\
\hline Basal & $0.60(0.05)$ & $0.28(0.04)$ & $0.32(0.05)$ & $3.0(0.1)$ & $37(2)$ & $32(2)$ \\
\hline $\begin{array}{l}\mathrm{IL}-1 \alpha(50 \mathrm{ng} / \mathrm{ml}) \\
\quad(\mathrm{n}=5)\end{array}$ & $\begin{array}{l}0.69(0.07)^{\mathrm{a}} \\
\Delta=0.09(0.03)\end{array}$ & $\begin{array}{l}0.30(0.04) \\
\Delta=0.02(0.01)\end{array}$ & $\begin{array}{l}0.38(0.05)^{\mathrm{a}} \\
\Delta=0.07(0.02)\end{array}$ & $2.7(0.1)^{\mathrm{a}}$ & $32(1)^{\mathrm{b}}$ & $30(2)$ \\
\hline Basal & $0.77(0.07)$ & $0.23(0.02)$ & $0.54(0.05)$ & $2.5(0.1)$ & $31(3)$ & $31(2)$ \\
\hline $\begin{array}{l}\mathrm{IL}-8(100 \mathrm{ng} / \mathrm{ml}) \\
\quad(\mathrm{n}=6)\end{array}$ & $\begin{array}{l}0.85(0.07)^{\mathrm{b}} \\
\Delta=0.07(0.01)\end{array}$ & $\begin{array}{l}0.26(0.02)^{\mathrm{a}} \\
\Delta=0.03(0.01)\end{array}$ & $\begin{array}{l}0.59(0.06)^{\mathrm{a}} \\
\Delta=0.05(0.02)\end{array}$ & $2.4(0.1)$ & 29 (3) & $30(2)^{\mathrm{a}}$ \\
\hline
\end{tabular}

Data are mean (SEM).

$\mathrm{J}_{\mathrm{ms}}$, mucosal to serosal flux; $\mathrm{J}_{\mathrm{sm}}$, serosal to mucosal flux; $\mathrm{J}_{\mathrm{net}}$, net flux; PD, potential difference; $\mathrm{I}_{\mathrm{sc}}$, short circuit current; $\mathrm{G}$, conductance. ${ }^{\mathrm{a}} \mathrm{p}<0.05,{ }^{\mathrm{b}} \mathrm{p}<0.01$ compared with basal period. 
Table 5 Effect of IL-8 on jejunal L-glucose transport

\begin{tabular}{|c|c|c|c|c|c|c|}
\hline & $\begin{array}{l}\mathcal{F}_{m s} \\
\left(\mu E q / \mathrm{cm}^{2} / h\right)\end{array}$ & $\begin{array}{l}\mathcal{F}_{s m} \\
\left(\mu E q / \mathrm{cm}^{2} / h\right)\end{array}$ & $\begin{array}{l}\mathcal{F}_{\text {net }} \\
\left(\mu E q / \mathrm{cm}^{2} / \mathrm{h}\right)\end{array}$ & $\begin{array}{l}P D \\
(m V)\end{array}$ & $I_{s c}\left(\mu A / \mathrm{cm}^{2}\right)$ & $\begin{array}{l}G \\
\left(\mathrm{mS} / \mathrm{cm}^{2}\right)\end{array}$ \\
\hline Basal & $0.186(0.042)$ & $0.216(0.035)$ & $-0.03(0.042)$ & $1.9(0.2)$ & $21(2)$ & $27(1)$ \\
\hline $\begin{array}{l}\mathrm{IL}-8(100 \mathrm{ng} / \mathrm{ml}) \\
(\mathrm{n}=5)\end{array}$ & $\begin{array}{l}0.194(0.029) \\
\Delta=0.08(0.05)\end{array}$ & $\begin{array}{l}0.232(0.044) \\
\Delta=0.02(0.03)\end{array}$ & $\begin{array}{l}-0.038(0.047) \\
\Delta=-0.01(0.07)\end{array}$ & $1.8(0.2)^{\mathrm{a}}$ & $18(2)^{b}$ & $25(1)^{a}$ \\
\hline
\end{tabular}

Data are mean (SEM).

$\mathrm{J}_{\mathrm{ms}}$, mucosal to serosal flux; $\mathrm{J}_{\mathrm{sm}}$, serosal to mucosal flux; $\mathrm{J}_{\text {net }}$, net flux; PD, potential difference; $\mathrm{I}_{\mathrm{sc}}$, short circuit current; $\mathrm{G}$, conductance. ${ }^{\mathrm{a}} \mathrm{p}<0.05,{ }^{\mathrm{b}} \mathrm{p}<0.01$ compared with basal period.

significant increase in the serosal to mucosal flux of L-proline.

\section{L-GLUCOSE TRANSPORT}

L-Glucose transport was examined to determine the effect of proinflammatory interleukins on passive glucose movement. The effect of IL-8 on jejunal L-glucose transport is shown in table 5 . Serosal IL-8 $(100 \mathrm{ng} / \mathrm{ml})$ had no effect on mucosal to serosal, serosal to mucosal, or net flux of L-glucose.

\section{$\left[{ }^{51} \mathrm{Cr}\right]$-EDTA PERMEABILITY}

The effect of IL- 8 on jejunal $\left[{ }^{51} \mathrm{Cr}\right]$-EDTA was also assessed to determine if proinflammatory interleukins altered 3-O-methyl glucose transport by changing passive tissue permeability. $\left[{ }^{51} \mathrm{Cr}\right]$-EDTA movement did not differ between basal and experimental periods in IL- 8 (basal $0.0018(0.0002) \quad v$ experimental 0.0015 $(0.0001) \%$ total hot $/ 5$ minutes; $n=4)$ or vehicle treated (basal $0.0026(0.0005) v$ experimental $0.0018(0.0001) ; \mathrm{n}=4)$ tissue.

TISSUE ELECTRICAL PARAMETERS

In the 3-O-methyl glucose transport experiments, all proinflammatory interleukins, except IL- $1 \alpha$ at its lowest concentration (5 $\mathrm{ng} / \mathrm{ml}$ ), caused a significant decrease in tissue PD (tables 1,2 ). $I_{s c}$ was also decreased in the presence of $50 \mathrm{ng} / \mathrm{ml} \mathrm{IL-} 1 \alpha, 10 \mathrm{ng} / \mathrm{ml} \mathrm{IL}-1 \alpha$,

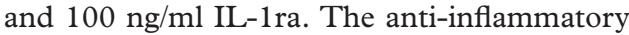
IL-10 had no effect on PD but resulted in a decrease in tissue $I_{s c}$. Tissue conductance did not differ in any of the 3-O-methyl glucose transport experimental groups. In the L-proline transport experiments, tissue PD was not affected by IL-1 $\alpha$ or IL-8 (table 4 ). However, both IL- $1 \alpha$, at a concentration of $50 \mathrm{ng} / \mathrm{ml}$, and IL-8 $(100 \mathrm{ng} / \mathrm{ml})$, resulted in a significant decrease in both $I_{s c}$ and conductance (G). Addition of $100 \mathrm{ng} / \mathrm{ml} \mathrm{IL-8}$ to the serosal surface in the L-glucose experiments resulted in a significant decrease in PD, $I_{s c}$, and G during the experimental period compared with basal values.

$\mathrm{Na}^{+} / \mathrm{K}^{+}$ATPase ACTIVITY

$\mathrm{Na}^{+} / \mathrm{K}^{+}$ATPase activity was measured in IL-8 and vehicle treated jejunal tissue to determine if proinflammatory interleukins alter the gradient for sodium entry into the enterocyte. $\mathrm{Na}^{+} / \mathrm{K}^{+}$ATPase activity did not differ between IL-8 (38.9 (5.7) U/mg protein; $n=6)$ and vehicle (36.2 (4.5); $\mathrm{n}=6)$ treated tissue.

SGLT-1 EXPRESSION

All BBMV preparations used in the study had a greater than 10-fold increase in sucrase activity compared with the original mucosal ho- mogenates and less than threefold basolateral contamination, as determined by $\mathrm{Na}^{+} / \mathrm{K}^{+}$ ATPase activity. BBMV protein content (control 7.06 (0.83); IL 7.42 (1.1) $\mathrm{mg} / \mathrm{ml} ; \mathrm{n}=8)$, sucrase activity (control 1169 (186); IL 1171 (172) $\mathrm{U} / \mathrm{g}$ protein; $\mathrm{n}=8$ ), and $\mathrm{Na}^{+} / \mathrm{K}^{+}$ATPase activity (control 95 (31); IL 147 (33) U/g protein; $n=8$ ) did not differ between control and interleukin (IL-8 or IL-6) treated tissue. Sucrase (control 99 (19); IL 99 (18) U/g protein; $\mathrm{n}=8$ ) and $\mathrm{Na}^{+} / \mathrm{K}^{+}$ATPase (control 171 (20); IL 165 (23) U/g protein; $n=8$ ) activity in the original mucosal homogenates also did not differ between control and interleukin (IL-8 or IL-6) treated tissue. Antibody staining revealed a single immunoreactive band at $\sim 75 \mathrm{kDa}$. SGLT-1 band density did not differ between BBMV harvested from control or IL- 8 treated tissue (control 3.30 (0.23); IL-8 2.82 (0.28) integrated optical density units; $n=4$ ) or control and IL- 6 treated tissue (control 4.96 (0.37); IL-6 4.31 (0.55) integrated optical density units; $n=4)$.

\section{Discussion}

The findings suggest that proinflammatory interleukins stimulate jejunal nutrient absorption. Anti-inflammatory interleukins appear to have no role in the regulation of jejunal nutrient transport.

Inflammation is a common response of the intestinal mucosa to pathological insults or injury. A variety of soluble mediators have been shown to participate in the gastrointestinal inflammatory process, including the proinflammatory interleukins IL- $1 \alpha,{ }^{23}$ IL- $6,{ }^{24}{ }^{25}$ and IL- $8,{ }^{26}$ the endogenous IL-1 receptor antagonist IL-1 $\mathrm{ra},{ }^{27}$ and the anti-inflammatory interleukin-10 (IL-10). ${ }^{28}$

Inflammation results in alterations in metabolic status. Elevated energy expenditure, enhanced gluconeogenesis, fat mobilisation, and protein catabolism are prominent features of inflammation induced hypermetabolism. ${ }^{8}{ }^{29}$ Furthermore, studies have suggested that the magnitude of the hypermetabolic response to tissue injury is directly proportional to the extent of tissue damage. ${ }^{30}$

In the current study, proinflammatory interleukins increased both glucose and proline uptake while the anti-inflammatory IL-10 had no effect. These findings suggest proinflammatory interleukins may produce a generalised increase in nutrient absorption, including hexose and amino acid uptake. Furthermore, this effect appears to be specific, as IL- $1 \alpha$ induced increases in glucose uptake were blocked in the presence of the IL-1 receptor antagonist, IL-1 ra. A number of studies have suggested a role for proinflammatory interleukins in the 
regulation of central and peripheral glucose metabolism. Interleukin- $1 \alpha$ has been reported to increase the uptake of glucose into both neonatal murine astrocytes ${ }^{14}$ and human dermal fibroblasts. ${ }^{13}$ In the former study, IL- 6 also increased glucose uptake into murine astrocytes; the small size of the effect reported may be due to the relatively low concentration of IL-6 used ( $2 \mathrm{ng} / \mathrm{ml}$ ) compared with concentrations used in our experiments. ${ }^{14}$ Intracerebroventricular IL-1 $\alpha$ has been shown to act centrally to increase whole body glucose metabolism and peripheral glucose utilisation. ${ }^{10}$ Intravascular IL-1 $\beta$ results in hypoglycaemia and altered whole body glucose homeostasis in mice. ${ }^{11}$ These findings are in agreement with our data demonstrating increased epithelial nutrient uptake in response to proinflammatory interleukins and suggest one of the roles of these cytokines is to increase nutrient availability to meet the increased metabolic demand of inflamed tissue.

All of the proinflammatory mediators examined in this study produced a consistent and significant decrease in tissue PD except IL- $1 \alpha$ at the lowest concentration tested $(5 \mathrm{ng} / \mathrm{ml})$. While there was a trend towards reduced $I_{s c}$ in all the experimental groups this effect only achieved significance in the IL- $1 \alpha(50 \mathrm{ng} / \mathrm{ml})$, IL- $1 \alpha+\mathrm{IL}-1 \mathrm{ra}$, and IL-10 experimental groups and in the L-glucose experiments. These findings are in contrast with a previous report on the effect of IL-1 $\alpha$ on ileal ion transport. Chiossone and colleagues ${ }^{3}$ measured an increase in short circuit current due to anion secretion. However, the effect reported by these authors was slow, reaching a maximum at 30-40 minutes. In contrast, our experiments were performed in jejunal tissue and flux measurements began five minutes following the addition of IL- $1 \alpha$ and continued for 15 minutes, thus ending after 20 minutes and well before the time point at which Chiossone et al reported their maximal effect. Furthermore, in our experiments $20 \mathrm{mM}$ 3-O-methyl glucose was added to the mucosal surface at mounting thus providing a substrate for SGLT-1. Addition of 3-O-methyl glucose activates SGLT-1 thus increasing enterocyte $\mathrm{Na}^{+}$uptake. This effect would tend to counteract the reported decrease in $\mathrm{Na}^{+}$and $\mathrm{Cl}^{-}$absorption seen by these authors. More recently, other investigators have examined the effect of IL-1 $\alpha$ on $\mathrm{Cl}^{-}$ secretion in T84 cells. Incubating T84 cells for 30-60 minutes with $5 \mathrm{ng} / \mathrm{ml}$ IL- $1 \alpha$ had no effect on basal $\mathrm{I}_{\mathrm{sc}} \cdot{ }^{31}$ As previously reported, ${ }^{5}$ IL-10 resulted in a significant decrease in tissue short circuit current.

Glucose can enter the bloodstream from the lumen of the intestine by two pathways: via a transcellular route across enterocyte cell membranes or by a paracellular route between epithelial cells. Transcellular glucose uptake occurs by an active uptake mechanism that couples the movement of glucose to the downhill gradient for sodium entry into the enterocyte. Glucose then exits the enterocyte via the facilitated glucose transporter GLUT2 and sodium by basolateral $\mathrm{Na}^{+} / \mathrm{K}^{+}$ATPase. ${ }^{32} \mathrm{Al}-$ terations in tissue $\mathrm{V}_{\max }$ may be attributed to changes in either the number of transport proteins, the rate at which the transporter translocates substrate across cell membranes, or in the chemical or electrical gradients driving the transport process. In adipocytes and striated muscle cells, glucose uptake is acutely increased in the presence of insulin due to recruitment of a pool of preformed transport proteins into the plasma membrane. ${ }^{33}$ Similarly, increases in jejunal glucose transport following administration of epidermal growth factor $^{34}$ or glucagon-like peptide $2^{35}$ have been associated with acute increases in the brush border content of the $\mathrm{Na}^{+} /$glucose cotransporter (SGLT-1). In the intestine, alterations in brush border membrane fluidity have been shown to alter rates of glucose and proline transport. ${ }^{36}$ Recently, a membrane associated protein, RS1, has been described which may have a role in the regulation of glucose transport. $^{38}$ Co-injection of RS1 cDNA together with SGLT-1 cDNA into oocytes results in a 40-fold increase in the activity of SGLT-1. The increased $\mathrm{V}_{\max }$ for jejunal glucose uptake observed in experimental type I diabetes is associated with an increase in $\mathrm{Na}^{+} / \mathrm{K}^{+}$ATPase protein and activity. ${ }^{39}$

Paracellular transport involves the movement of glucose between enterocytes in the epithelial layer either by passive diffusion or solvent drag. ${ }^{40}$ Paracellular transport can be modulated by a variety of stimuli all with a common effector mechanism apparently tied to the cytoskeleton. ${ }^{40}$ Activation of $\mathrm{Na}^{+}$couple glucose transport has been reported to elicit alterations in intestinal epithelial tight junctions leading to increased paracellular permeability and passive solute uptake. ${ }^{41}{ }^{42}$ Paracellular permeability has also been shown to be modulated by external agents, such as interferon $\gamma^{43}$ and intracellular mediators such as protein kinase C. ${ }^{44}$ However, in our studies jejunal glucose transport was assessed in short circuited Ussing chambers. This experimental set up eliminates all passive electrical and chemical gradients. Thus the increase in jejunal glucose transport seen following administration of proinflammatory interleukins is unlikely to have been caused by any change in passive tissue permeability. To confirm that altered permeability was not a factor, the epithelial movement of L-glucose and Cr-EDTA was examined. Treatment with IL-8 also had no effect on passive L-glucose or Cr-EDTA movement, further supporting the interpretation that proinflammatory interleukins increase glucose transport by an active mechanism. There was a small increase in the serosal to mucosal flux that reached statistical significance in many of the treatment groups. This likely reflects a small increase in paracellular permeability and is probably due to a specific action of the cytokines. Serosal to mucosal flux did not significantly differ in vehicle treated control experiments or in studies examining the effect of concurrent IL- $1 \alpha$ and the antagonist IL-1 ra.

In the current study, $\mathrm{Na}^{+} / \mathrm{K}^{+}$ATPase activity did not differ between mucosal homogenates obtained from control or IL-8 treated tissue 
suggesting that interleukin induced increases in glucose transport are not due to alterations in the transcellular sodium gradient. Furthermore, the increase in jejunal glucose transport seen following exposure to proinflammatory cytokines does not appear to be due to alterations in the brush border expression of SGLT-1. The lack of alteration in either $\mathrm{Na}^{+} / \mathrm{K}^{+}$ ATPase or SGLT-1 suggests the possibility that proinflammatory interleukins increase jejunal glucose transport by modulating the efficiency of the existing population of $\mathrm{Na}^{+} /$glucose cotransporters.

Inflammatory mediators may be derived from mononuclear cells in the lamina propria or from epithelial cells, or both. ${ }^{12326-28} 4546$ The site of cytokine production can vary depending on the inflammatory stimulus. For example, epithelial cells in normal and inflamed tissue express neither IL-8 mRNA nor protein. IL-8 is produced primarily in the lamina propria of the colon in inflammatory bowel disease. IL-8 mRNA and protein are localised to macrophages and neutrophils near the site of ulceration. ${ }^{46}$ In contrast, infection of T 84 monolayers with $S$ typhimurium induces the synthesis and release of IL-8. ${ }^{47}$ Similarly, Clostridium difficile toxin $\mathrm{A}$ induces the production of IL-8 from human colonic epithelial cells. ${ }^{48}$ IL-6 is primarily produced by macrophages and monocytes, fibroblasts, and endothelial cells. ${ }^{1}$ $S$ typhimurium infection can induce the production of IL- 6 from small and large intestinal epithelial cells. ${ }^{45}$ Thus the site of production of bioactive cytokines varies depending on the site and mechanism of injury and the effect of proinflammatory interleukins on intestinal nutrient transport may be the result of endocrine, paracrine, or autocrine activity.

It is not known whether the cytokines used in this study are acting on the epithelium directly or if they are acting through release of other mediators from cellular elements in the intestinal wall. Experiments performed with the nerve blocker tetrodotoxin suggest enteric nerves do not play a role in mediating the effect of the proinflammatory interleukins on nutrient transport. Type I IL-1 receptor protein and mRNA have been associated with small intestinal epithelial cells. ${ }^{49}{ }^{50}$ IL- 6 receptors have been reported on colonic epithelial cells. ${ }^{51}$ In addition, functional receptors for a variety of other interleukins have also been described on intestinal epithelial cells, including those for IL-2, IL-4, IL-7, and IL-13..$^{52-55}$ These findings indicate that the effect of these proinflammatory cytokines on small intestinal nutrient transport may be due to a direct action on intestinal epithelial cells.

During the inflammatory process pro- and anti-inflammatory mediators are produced and released in the intestinal mucosa at concentrations similar to those used in our experiments. Concentrations of IL-6 released from $S$ typhimurium infected small intestinal cell cultures exceeded $10 \mathrm{ng} / \mathrm{ml}^{45}$ and biopsies obtained from Crohn's patients contained IL- 6 at concentrations greater than $15 \mathrm{ng} / \mathrm{ml} .^{24}$ Bacterial invasion of T84, HT29, and Caco-2 cell monolayers, as well as freshly isolated human colonic epithelial cells, induces the release of IL-8 into the media in concentrations ranging from 1.4 to $80.5 \mathrm{ng} / \mathrm{ml} .^{56}$ Moreover, IL-10 modulates ion transport in rat small intestine with a maximal response at a concentration of $1 \mathrm{ng} / \mathrm{ml}^{5}$ and serosal IL- $1 \alpha$ produces its maximal effect on rabbit ileal mucosal ion transport at a concentration of $5 \mathrm{ng} / \mathrm{ml} .^{3}$ In our study the effect of IL- $1 \alpha$ on nutrient uptake was also maximal at a concentration of $5 \mathrm{ng} / \mathrm{ml}$. Therefore, the effect of proinflammatory mediators on intestinal nutrient uptake occurs within the physiological range of these compounds that are released during disease states.

In summary, our findings suggest that release of proinflammatory interleukins promotes the uptake of nutrients across the small intestinal epithelium. The enhanced uptake of nutrients may play a role in the intestinal response to the hypermetabolic state observed during inflammatory episodes.

This work was supported by the Medical Research Council of Canada.

1 Cohen MC, Cohen S. Cytokine function: a study in biologic diversity. Am f Clin Pathol 1996;105:589-98.

2 Sartor RB. Cytokines in intestinal inflammation: pathophysiological and clinical considerations. Gastroenterology 1994;106:533-9.

3 Chiossone DC, Simon PL, Smith PL. Interleukin-1: effects on rabbit ileal mucosal ion transport in vitro. Eur $f$ Pharmacol 1990;180:217-28.

4 Chang EB, Musch MW, Mayer L. Interleukins 1 and 3 stimulate anion secretion in chicken intestine. Gastroenterology 1990;98:1518-24.

5 Madsen KL, Tavernini MM, Mosmann TR, Fedorak RN. Madsen KL, Tavernini MM, Mosmann TR, Fedorak RN. Interleukin 10 modulates ion transport

6 Madsen KL, Lewis SA, Tavernini MM, Hibbard J, Fedorak $\mathrm{RN}$. Interleukin 10 prevents cytokine-induced disruption of T84 monolayer barrier integrity and limits chloride secretion. Gastroenterology 1997;113:151-9.

7 Hiribarren A, Heyman M, L'Helgouac'h A, Desjeux JF. Effect of cytokines on the epithelial function of the human colon carcinoma cell line HT29 cl 19A. Gut 1993;34:61620.

8 Staal-van den Brekel AJ, Dentener MA, Schols AMWJ, Buurman WA, Wouters EFM. Increased resting energy expenditure and weight loss are related to a systemic inflammatory response in lung cancer patients. F Clin Oncol 1995;13:2600-5.

9 Lennie TA, McCarthy DO, Keesey RE. Body energy status and the metabolic response to acute inflammation. $A m \mathcal{F}$ Physiol (Regul Integr Comp Physiol) 1995;269:R1024-31.

10 Lang CH, Molina PE, Yousef KA, Tepper PG, Abumrad NN. Role of IL- $1 \alpha$ in central nervous system immunomodulation of glucoregulation. Brain Res 1993;624:5360

11 Del Rey A, Besedovsky H. Interleukin 1 affects glucose homeostasis. Am $\mathcal{f}$ Physiol (Regul Integr Comp Physiol) 1987;253:R794-8.

12 Berridge MV, Tan AS. Interleukin-3 facilitates glucose transport in a myeloid cell line by regulating the affinity of the glucose transporter for glucose: involvement of protein phosphorylation in transporter activation. Biochem $\mathcal{F} 1995$; 305:843-51

13 Taylor DJ, Faragher EB, Evanson JM. Inflammatory cytokines stimulate glucose uptake and glycolysis but reduce glucose oxidation in human dermal fibroblasts in vitro. Cir Shock 1992;37:105-10.

$14 \mathrm{Yu} \mathrm{N}$, Maciejewski-Lenoir D, Bloom FE, Magistretti PJ. Tumor necrosis factor- $\alpha$ and interleukin- $1 \alpha$ enhance glucose utilization by astrocytes: involvement of phospholiglucose utilization by astrocytes: involve
pase A . Mol Pharmacol 1995;48:550-8.

15 Clarkson TW, Toole SR. Measurement of short-circuit current and ion transport across the ileum. Am f Physiol 1964; 206:658-68.

16 Lowry OH, Rosebrough NJ, Farr AL, Randall RJ. Protein measurement with the Folin phenol reagent. $f$ Biol Chem 1951;193:265-75.

17 Kelly M, Butler DG, Hamilton JR. Transmissable gastroenteritis in piglets: a model of infantile viral diarrhea. $\mathcal{F}$ Pediatr 1972;80:925-31.

18 Kessler M, Acuto O, Storelli C, Murer H, Muller M, Semenza G. A modified procedure for the rapid preparation of efficiently transporting vesicles from small intestinal brush border membranes. Their use in investigating some
properties of D-glucose and choline transport systems. Bioproperties of D-glucose and choline
chim Biophys Acta 1978;771:35-41.

19 Dalquist A. Method for assay of intestinal dissacharidases. Anal Biochem 1964;7:18-25. 
20 Laemmli UK. Cleavage of structural proteins during the assembly of the head of the bacteriophage T Nature 1970 227:680-5.

21 Hirayama BA, Wong HC, Smith CD, Hagenbuch BA, Hediger MA, Wright EM. Intestinal and renal $\mathrm{Na}^{+} /$glucose cotransporters share common structures. Am f Physiol (Cell Physiol) 1991;261:C296-304.

22 Fowler SJ. The detection of proteins on blots using gold or immunogold. In: Walker JM, ed. Methods in molecular biology. Totowa, NJ: Humana Press, 1994:239-55.

23 Youngman KR, Simon PL, West GA, et al. Localization of intestinal interleukin 1 activity and protein and gene expression to lamina propria cells. Gastroenterology 1993; 104:749-58.

24 Reimund J-M, Wittersheim C, Dumont S, et al. Increased production of tumor necrosis factor- $\alpha$, interleukin- $1 \beta$, and interleukin- 6 by morphologically normal intestinal biopsies from patients with Crohn's disease. Gut 1996;39:684-9.

25 Hyams JS, Fitzgerald JE, Treem WR, Wyzga N, Kreutzer DL. Relationship of functional and antigenic interleukin 6 to disease activity in inflammatory bowel disease, Gastroen to disease activity in inflammat

26 Daig R, Andus T, Aschenbrenner E, Falk W, Scholmerich J, Gross V. Increased interleukin 8 expression in the colon mucosa of patients with inflammatory bowel disease. Gut 1996;38:216-22.

27 Cominelli F, Pizarro TT. Interleukin-1 and interleukin-1 receptor antagonist in inflammatory bowel disease. Aliment Pharmacol Ther 1996;10(suppl 2):49-53.

28 Nielsen OH, Koppen T, Rudiger N, Horn T, Eriksen J, Kirman I. Involvement of interleukin-4 and -10 in inflammatory bowel disease. Dig Dis Sci 1996;41:1786-93.

29 Ling PR, Bistrian BR, Mendez B, Istfan NW. Effects of systemic infusions of endotoxin, tumor necrosis factor, and interleukin-1 on glucose metabolism in the rat: relationship to endogenous glucose production and peripheral tissue glucose uptake. Metabolism 1994;43:279-84.

30 Wilmore DW. Nutrition and metabolism following thermal injury. Clin Plast Surg 1974;1:603-19.

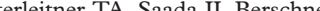
Valentich JD. IL-1 stimulates intestinal myofibroblast COX gene expression and augments activation of $\mathrm{Cl}^{-}$secretion in gene expression and augments activation of $\mathrm{Cl}^{-}$secretion in

32 Wright EM, Hirayama BA Lar K Intestinal sugar transport. In: Johnson LR, ed. Physiology of the gastrointestinal tract. New York: Raven Press, 1994 $1751-72$.

33 Holman GD, Cushman SW. Subcellular localization and trafficking of the GLUT4 glucose transporter isoform in insulin-responsive cells. Bioessays 1994;16:753-9.

34 Chung BM, Wong JK, Hardin JA, Gall DG. SGLT1 expression and the role of actin in EGF-induced alterations in enterocyte membrane function and surface area. $A m \mathcal{F}$ Physiol (Gastrointest Liver Physiol) 1999;276:G463-9.

35 Cheeseman CI. Upregulation of SGLT-1 transport activity in rat jejunum induced by GLP-2 infusion in vivo. $A m \mathcal{F}$ Physiol (Regul Integrative Comp Physiol) 1997;273:R196571 .

36 Meddings JB, DeSouza D, Goel M, Theisen S. Glucose transport and microvillus membrane physical properties 85:1099-107.

37 Sadowski DC, Gibbs DJ, Meddings JB. Proline transport across the intestinal microvillus membrane may be regulated by membrane physical properties. Biochim Biophys Acta 1992;1105:75-83.
38 Veyhl M, Spangenberg J, Puschel B, et al. Cloning of a membrane-associated protein which modifies activity and properties of the $\mathrm{Na}^{+}$-D-glucose cotransporter. $7 \mathrm{Biol}$ Chem 1993:268:25041-53.

39 Barada K, Okolo C, Field M, Cortas N. Na,K-ATPase in diabetic rat small intestine: changes at protein and mRNA levels and role of glucagon. F Clin Invest 1994;93:2725-31.

40 Madara JL. Regulation of the movement of solutes across tight junctions. Annu Rev Physiol 1998;60:143-59.

41 Atisook K, Carlson S, Madara JL. Effects of phlorizin and sodium on glucose-elicited alterations of cell junctions in intestinal epithelia. Am f Physiol 1990;258:C77-85.

42 Pappenheimer JR. Physiological regulation of epithelial junctions in intestinal epithelia. Acta Physiol Scand Suppl 1988;571:43-51.

43 Madara JL, Stafford J. Interferon-gamma directly affects barrier function of cultured intestinal epithelial monolayers. $\mathcal{F}$ Clin Invest 1989;83:724-7.

44 Stenson WF, Easom RA, Riehl TE, Turk J. Regulation of paracellular permeability in Caco-2 cell monolayers by protein kinase C. Am F Physiol (Gastrointest Liver Physiol) 1993;265:G955-62.

45 Weinstein DL, O'Neill BL, Metcalf ES. Salmonella typhi stimulation of human intestinal epithelial cells induces secretion of epithelial cell-derived interleukin- 6. Infect Immun 1997;65:395-404.

46 Grimm MC, Elsbury SKO, Pavli P, Doe WF. Interleukin 8: cells of orgin in inflammatory bowel disease. Gut 1996;38: 90-8.

47 McCormick BA, Colgan SP, Delp-Archer C, Miller SI, Madara JL. Salmonella typhimurium attachment to human intestinal epithelial monolayers: transcellular signalling to subepithelial neutrophils. $\mathcal{F}$ Cell Biol 1993;123:895-907.

48 Branka J-E, Vallette G, Jarry A, et al Early functional effects of Clostridium difficile toxin A on human colonocytes. Gastroenterology 1997;112:1887-94.

49 McGee DW, Vitkus SJD, Lee PY. The effect of cytokine stimulation on IL-1 receptor mRNA expression by intestinal epithelial cells. Cell Immunol 1996;168:276-80.

50 Sutherland DB, Varilek GW, Neil GA. Identification and characterization of the rat intestinal epithelial cell (IEC-18) nterleukin-1 receptor. Am f Physiol (Cell Physiol) 1994; 266:C1198-203 (abstract).

51 Shirota K, LeDuy L, Yuan S, Jothy S. Interleukin-6 and its receptor are expressed in human intestinal epithelial cells. Virchows Arch B Cell Pathol 1990;58:303-8.

52 Dignass AU, Podolsky DK. Interleukin 2 modulates intestinal epithelial cell function in vitro. Exp Cell Res 1996;225: 422-9.

53 Yamada K, Shimaoka M, Nagayama K, Hiroi T, Kiyono H, Honda T. Bacterial invasion induces interleukin-7 receptor expression in colonic epithelial cell line, T84. Eur f Immunol 1997;27:3456-60 (abstract).

54 Zund G, Madara JL, Dzus AL, Awtrey CS, Colgan SP. Interleukin-4 and interleukin-13 differentially regulate epithelial chloride secretion. 7 Biol Chem 1996;271:7460-4 (abstract)

55 Colgan SP, Resnick MB, Parkos CA, et al. IL-4 directly modulates function of a model human intestinal epithelium. F Immunol 1994;153:2122-9 (abstract).

56 Jung HC, Eckmann L, Yang S-K, et al. A distinct array of proinflammatory cytokines is expressed in human colon epithelial cells in response to bacterial invasion. $f$ Clin Invest 1995;95:55-65. 